\title{
Do Various Treatment Modalities of Vesicoureteral Reflux Have Any Adverse Effects in Pediatric Patients? A Meta-Analysis
}

\author{
Min Xie $^{a} \quad X_{i a o g a i} \mathrm{Xu}^{\mathrm{b}}$ Zhenjie Cao ${ }^{c}$ Huijie Xiao ${ }^{\mathrm{d}}$ \\ aDepartment of Pediatric Nephrology, Chengdu Women's and Children's Central Hospital, School of Medicine, \\ University of Electronic Science and Technology of China, Chengdu, China; ${ }^{\text {b} D e p a r t m e n t ~ o f ~ N e u r o l o g y, ~ C h i l d r e n ' s ~}$ \\ Hospital of Shanxi (Women Health Center of Shanxi), Taiyuan, China; 'Pediatric Surgery, The Third Affiliated Hospital of

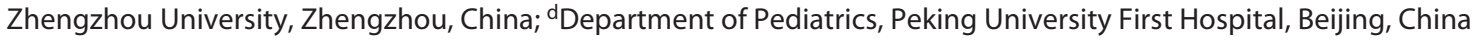

\section{Keywords}

Vesicoureteral reflux · Urinary tract infections · Renal scar · Meta-analysis

\begin{abstract}
Purpose: Vesicoureteral reflux (VUR) is a risk factor for various renal problems like recurrent urinary tract infections (UTIs), pyelonephritis, renal scarring, hypertension, and other renal parenchymal defects. The interventions followed by pediatricians include low-dose antibiotic treatment, surgical correction, and endoscopy. This meta-analysis aimed to assess the advantages and drawbacks of various primary VUR treatment options. Search Strategy: The Cochrane Central Register of Controlled Trials, MEDLINE, EMBASE, reference lists of journals, and abstracts from conference proceedings were all used to find randomized controlled trials. The articles were retrieved from 1985 till 2020. Twenty articles were used for the data analysis. Criteria for Selection: Surgery, long-term antibiotic prophylaxis, noninvasive techniques, and any mix of therapies are also options for treating VUR. Collection and Interpretation of Data: Two authors searched the literature separately, determining research qualifications, assessing accuracy, and extracting and entering results. The odds ratio $(O R)$ of these studies was used to construct the forest plot. The random-effects model was
\end{abstract}

used to pool the data. Also, the random-effects model was used with statistical significance at a $p$ value $<0.05$ to assess the difference in side effects after treatment of VUR using different modalities. Results: We found no statistically significant differences between surgery plus antibiotics and antibiotic alone-treated patients in terms of recurrent UTIs (OR $=0.581 ; 95 \%$ confidence interval $[\mathrm{Cl}] 0.259-1.30)$, renal parenchymal defects $(\mathrm{OR}=1.149 ; 95 \% \mathrm{Cl} 0.75-1.754)$, and renal scarring $(\mathrm{OR}=1.042 ; 95 \% \mathrm{Cl} 0.72-1.50)$. However, the risk of developing pyelonephritis after surgical treatment of VUR was lesser than that in the conservative approach, that is, antibiotics (OR $=0.345 ; 95 \% \mathrm{Cl} 0.126-0.946$.), positive urine culture $(\mathrm{OR}=0.617 ; 95 \% \mathrm{Cl} 0.428-0.890)$, and recurrent UTIs were more common in the placebo group than in the antibiotic group ( $p<0.05$; OR $=0.639 ; 95 \% \mathrm{Cl} 0.436-0.936$ ) which is statistically significant. Conclusion: Based on current research, we recommend that a child with a UTI and significant VUR be treated conservatively at first, with surgical care reserved for children who have issues with antimicrobials or have clinically significant VUR that persists after several years of follow-up.

(c) 2021 The Author(s)

Published by S. Karger AG, Basel

Min Xie and Xiaogai Xu are both the first authors, they contributed equally.
(C) 2021 The Author(s)

Published by S. Karger AG, Basel

This is an Open Access article licensed under the Creative Commons Attribution-NonCommercial-4.0 International License (CC BY-NC) (http://www.karger.com/Services/OpenAccessLicense), applicable to the online version of the article only. Usage and distribution for commercial purposes requires written permission.
Correspondence to:

Huijie Xiao, huijiexiao521@ sina.com 


\section{Introduction}

Urinary tract infection (UTI) is the most common infection among children $[1,2]$. One of the most common causes of problems related to the urinary tract among children is vesicoureteral reflux (VUR). VUR is the retrograde flow of the urine from the bladder to the ureter and renal pelvis [3]. Previous studies have shown that this is one of the most common predisposing factors for UTI. UTI combined with VUR is a risk factor for various renal problems like pyelonephritis, renal scarring, hypertension, and other renal parenchymal defects [4]. However, there is debate on the causal association between these entities, and data on the actual occurrence of clinically relevant VUR complications are scarce [3]. Due to the lack of objective data, most VUR management assessments have been based on expert views [4], and the superiority of either medical or surgical treatment in avoiding kidney damage has not been shown [5-8].

Although the precise incidence in the general infant population is uncertain, VUR is consistently observed in around a third of children with UTIs. In addition, UTI affects $5-10 \%$ of girls, while VUR affects $1-3 \%$ [2]. Thus, VUR is thought to predispose sufferers to UTIs involving the kidney material, leading to permanent renal damage. While recent findings from a prospective cohort study suggest that adverse effects of renal damage associated with UTI are significantly lower, retrospective analyses of selected patients with renal scarring report hypertension in about $20 \%$ of patients and chronic kidney disease $10 \%$ of patients [4-8].

The prevention of UTI-induced disruption has become the primary treatment technique for children with VUR. Surgical reflux correction and long-term antibiotic prophylaxis, either alone or in combination, have been tried. Latest, less intrusive procedures such as endoscopic periureteric injections of polydimethylsiloxane (Macroplastique), dextranomer/hyaluronic acid copolymer (Deflux), or glutaraldehyde cross-linked bovine collagen have been evaluated in addition to the traditional Politano-Leadbetter and Cohen surgical techniques [9-12].

Because VUR is a widespread childhood issue, there is much debate over the proper treatment. The Pediatric Vesicoureteral Reflux Guidelines Panel [1] published a study that resulted in few clinical suggestions focused on empirical proof of efficacy, instead of focusing on "panel advice" for most decisions.

\section{Rationale}

Due to a lack of scientific data, management decisions on VUR have primarily been focused on expert views, and the superiority of either medical or surgical treatment in avoiding kidney damage has not been shown. We used renal parenchymal defects, renal scarring, pyelonephritis, and recurrence of UTI as endpoints in a meta-analysis to evaluate surgical and conservative treatment effectiveness, that is, the prevalence of side effects. We tried to see if any research could help a clinician choose between these treatment options. This research aimed to assess the evidence for the advantages and risks of the currently available treatment methods, including operative, nonoperative, and no intervention.

\section{Objective}

The objective of this study was to assess the data for the advantages and drawbacks of various key VUR care options.

\section{Materials and Methods}

We followed the PRISMA normative recommendations in this study with the registration number PU \# RC/IRB/2020/1036.

\section{Eligibility Criteria for Considering Studies}

Any therapy in primary VUR was tested in randomized controlled trials (RCTs) and quasi-RCTs (RCTs in which allocation of care was achieved through alternation, alternative medical history, date of birth, or other predictable methods). The articles were reviewed from the title or abstract. Case reports, solely neonatal or adult studies, reviews, editorial commentaries, animal studies, articles about secondary or sibling VUR, and publications concerned exclusively with UTI were excluded. The articles concerned only with children, with at least one defined treatment group, addressed the various side effects, and written in English with no other limiting factor were used in finalizing the studies.

\section{Data Search}

The authors searched the medical literature on the topic was extensively searched in MEDLINE, EBSCO, and UPTODATE databases from 1985 to 2020. There was no restriction on the selection of articles based on their year of publication. The author's used the MESH terms to search for the topic. The keywords used were urogenital abnormalities, VUR, renal parenchymal defects, UTIs, renal scarring, pyelonephritis, antibiotic, surgical, and endoscopy.

Selection Process

Information on the following aspects was included in the metaanalysis:

- Recurrent UTI after use of antibiotics compared to a placebo group

- Recurrent UTI after endoscopic surgery compared to antibiotics

- Positive urine culture after surgery and antibiotics compared to administration of antibiotics alone 
- Renal parenchymal defects after endoscopic surgery compared to antibiotics

- Renal scarring after endoscopic surgery compared to antibiotics

- Pyelonephritis after endoscopic surgery compared to antibiotics The primary search yielded 10,355 results. Articles were debarred based on the exclusion criteria. These included 57 articles, out of which 20 articles were included in data analysis. The selection of articles was made after critical evaluation by a panel of 3 experts. They were given a vital evaluation checklist for the article review. The final selection was based on the review by a panel of experts. Details of the number of articles included are given in Figure 1.

At least 3 reviewers independently screened abstracts received from the above searches for selection (M.X., X.X., and H.X.). Any discrepancies were worked out with the help of a fourth author (Z.C.). When the suitability of an essay was questioned, or there was no abstract available, the entire article was retrieved. Over selection was preferred in all situations to prevent applicable missing studies and ensure that reference lists could find additional studies. Where applicable, authors were contacted to collect raw or incomplete data.

A random-effects model was used to obtain overall estimates of event probabilities (e.g., recurrent UTIs, renal scars, renal parenchymal defects, etc.). After tabulating the data, individual study estimates were obtained with the overall forecast and observation characteristics using the odds ratio (OR). Differences between or among specific groups were assessed using the $Z$ test. Statistical significance was set at 0.05 for comparing the 2 groups. Forest plots were used to represent individual study estimates (points) and their 95\% confidence intervals (CIs), together with the overall assessment. The meta-analysis was carried out using Comprehensive Meta-Analysis Software Version 3.

\section{Results}

\section{Literature Search}

The primary search yielded 10,355 results. Articles were debarred based on the exclusion criteria. These included 57 articles, out of which 20 articles were included in data analysis. The selection of articles was made after critical evaluation by a panel of 3 experts. They were given a vital evaluation checklist for the article review. The final selection was based on the review by a panel of experts. Details of the included studies are given in Table 1.

\section{Risk of Bias}

Five studies found the treatment distribution process to be satisfactory. Just 2 analyses found that radiological results were assessed without knowing which treatment groups they were in. In 4 trials, there was no intention-totreat analysis; in the other studies, it was unable to decide if the analysis was conducted on an intention-to-treat basis. Losses due to follow-up were generally poor, ranging between 0 and 2\% after $1-2$ years and 9 and $42 \%$ after 4-10 years.

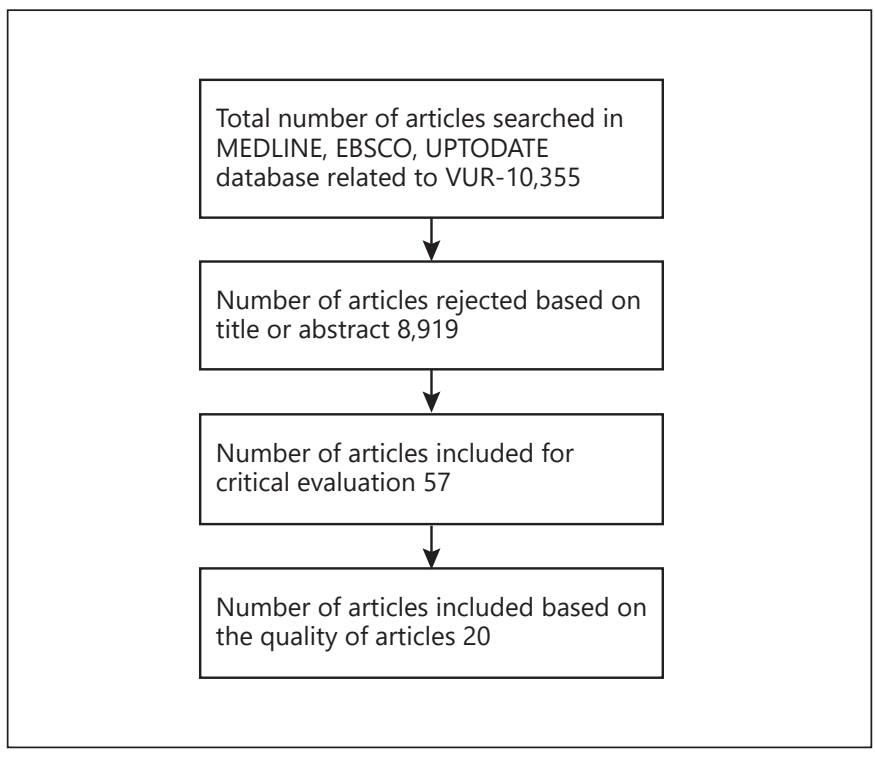

Fig. 1. The details of the number of articles included.

\section{Effects of Interventions}

Recurrent UTI in Placebo versus Antibiotic Group

A total number of 8 studies were evaluated for the odds of developing recurrent UTIs in the treatment of VUR among pediatric patients with the help of a random-effects model. Cumulative statistics were significant at a $95 \%$ confidence level with $p<0.05(0.021)$. On the left side, it favors treatment with antibiotics alone with a CI varying from 0.436 to 0.936 with an OR of 0.639 , indicating that developing recurrent UTIs after antibiotics treatment for VUR was 0.639 times more than that in the placebo group (Fig. 2).

Positive Urine Culture after Surgery and Antibiotics Compared to Administration of Antibiotics Alone

A total of 7 studies were assessed for the odds of developing a positive urine culture between the 2 treatment modalities. Cumulative statistics were significant at a $95 \%$ confidence level with $p<0.05$ (0.010). The cumulative statistics on the left side favor a combined approach of surgery and antibiotics compared to antibiotics alone, with an OR of 0.617 which indicates that a positive urine culture is more likely to develop in the surgery and antibiotics approach than antibiotics alone (Fig. 3).

\section{Renal Parenchymal Defects after Endoscopic Surgery}

Compared to Antibiotics

Similarly, a total number of 4 studies were examined for evaluating the odds of developing renal parenchymal 


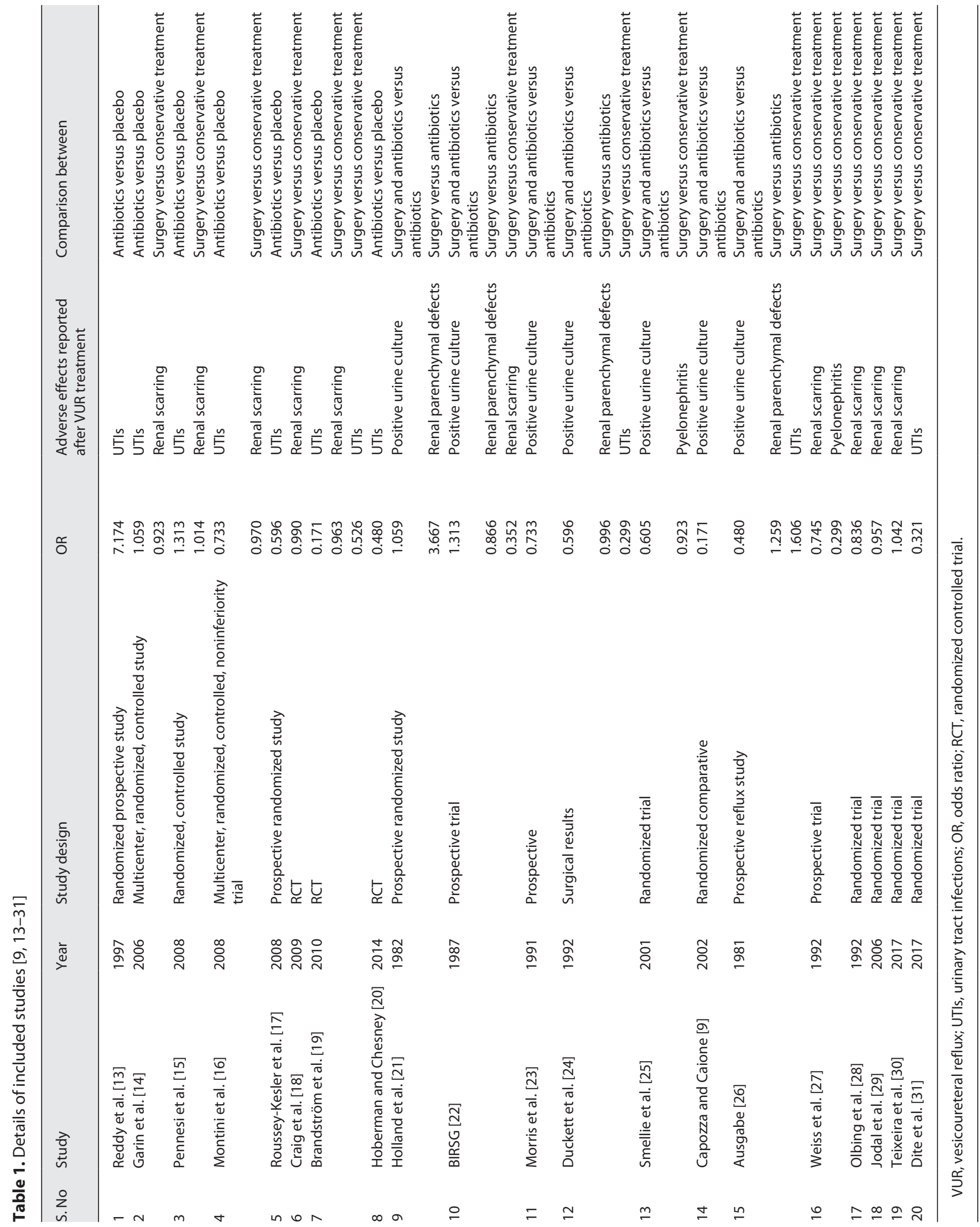




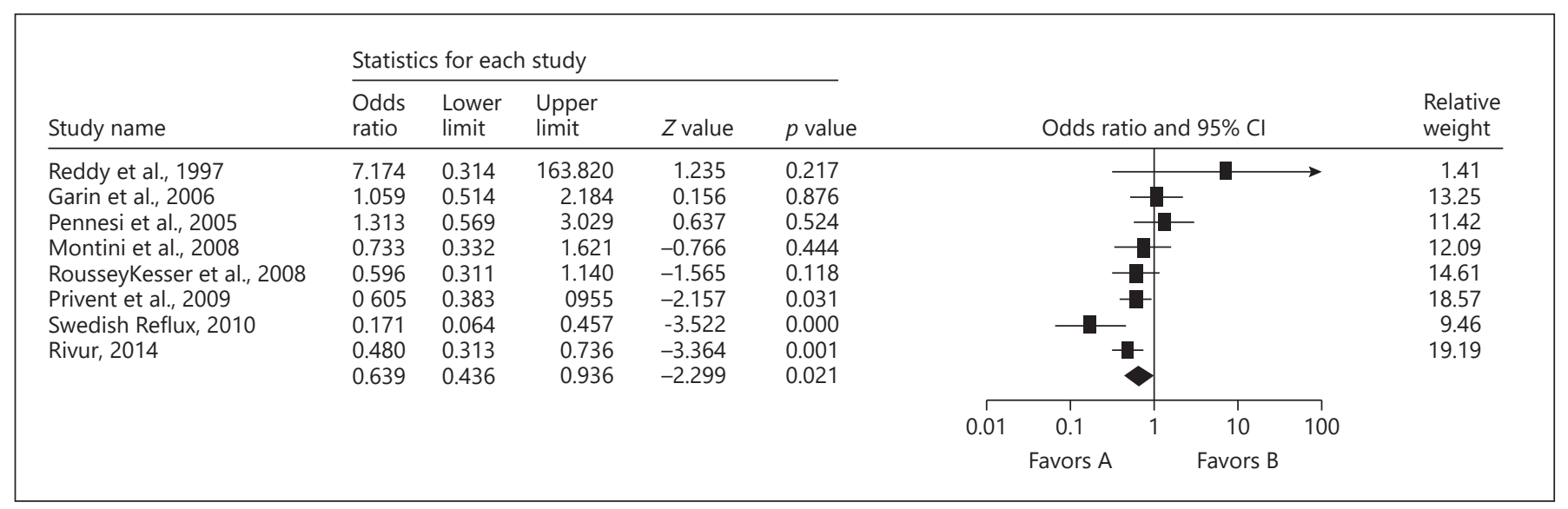

Fig. 2. Shows the forest plot between the chances of recurrent UTIs in comparison of antibiotics with placebo treatment for VUR. VUR, vesicoureteral reflux; OR, odds ratio; UTIs, urinary tract infections.

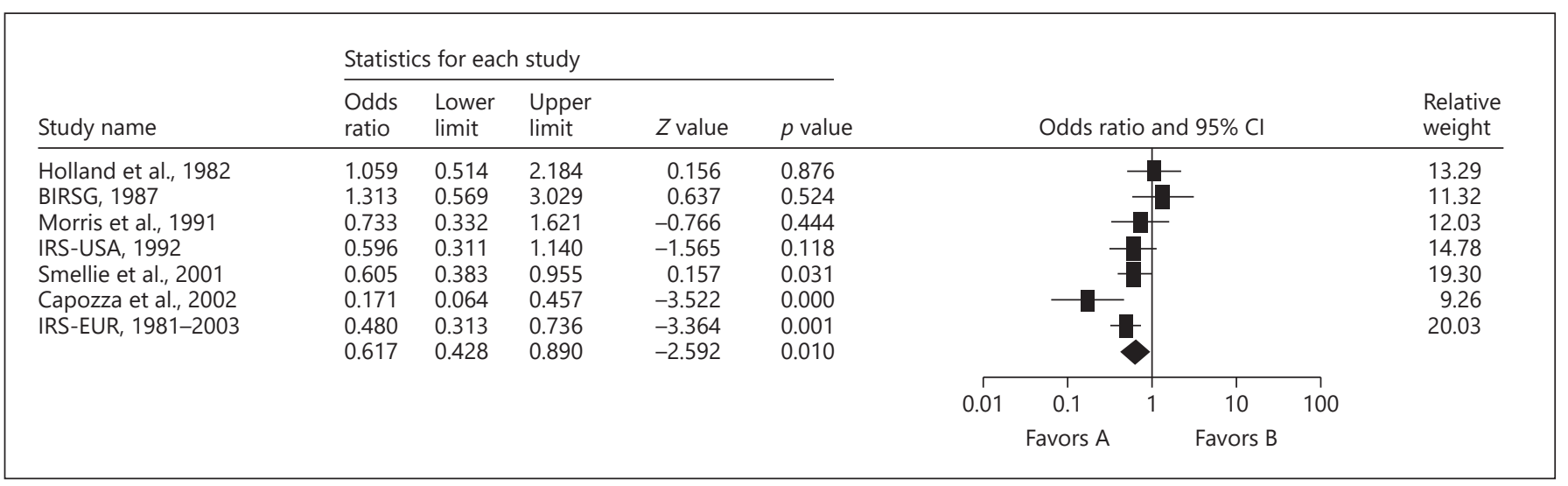

Fig. 3. Shows the forest plot for the positive urine culture report after treating VUR with a combination of surgery and antibiotics as compared to antibiotics alone. VUR, vesicoureteral reflux; OR, odds ratio.

defects in the 2 groups of surgery compared to antibiotics. Again, cumulative statistics were not significant at 95\% confidence level with $p>0.05$ (0.517), that is, there is no statistically significant difference existing in both treatment modalities for renal parenchymal defects with an OR of 1.149 which indicates that renal parenchymal defects are more likely to develop in the groups treated with surgery than antibiotics (Fig. 4).

\section{Renal Scarring after Endoscopic Surgery Compared} to Antibiotics

A total number of 4 studies were assessed for evaluating the odds of developing renal scarring among the 2 groups, that is, surgery versus no surgery. The cumulative statistics were not statistically significant at a $95 \%$ confidence level with $p>0.05(0.827)$, that is, there is no dif- ference in both the treatment modalities on renal scarring with an OR of 1.042, indicating that renal scarring is more likely to be present in the groups treated with surgery than conservative treatment (Fig. 5).

\section{Recurrent UTI after Endoscopic Surgery Compared} to Antibiotics

A total number of 4 studies were examined for evaluating the odds of developing recurrent UTIs among the groups, that is, surgery versus no surgery. The cumulative statistics were not statistically significant at a $95 \%$ confidence level with $p>0.05(0.189)$, that is, there is no difference in both the treatment modalities for UTIs with an OR of 0.581 which indicates that UTIs are more likely to be present in the groups treated with surgery than conservative treatment (Fig. 6a). 


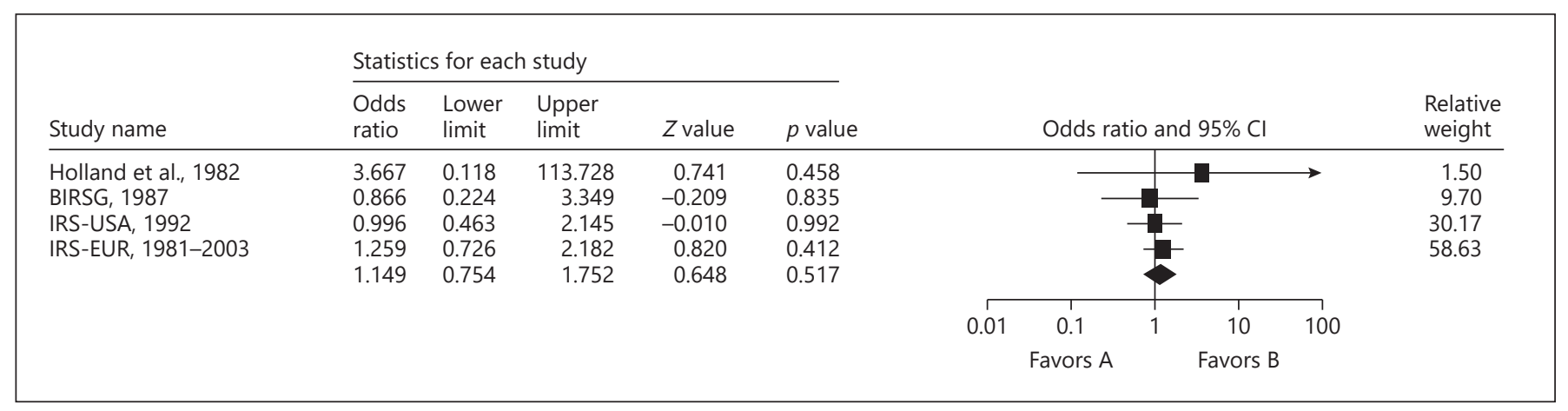

Fig. 4. Shows the forest plot for the renal parenchymal defects developed after treating VUR with surgery as compared to antibiotics alone. VUR, vesicoureteral reflux; OR, odds ratio.

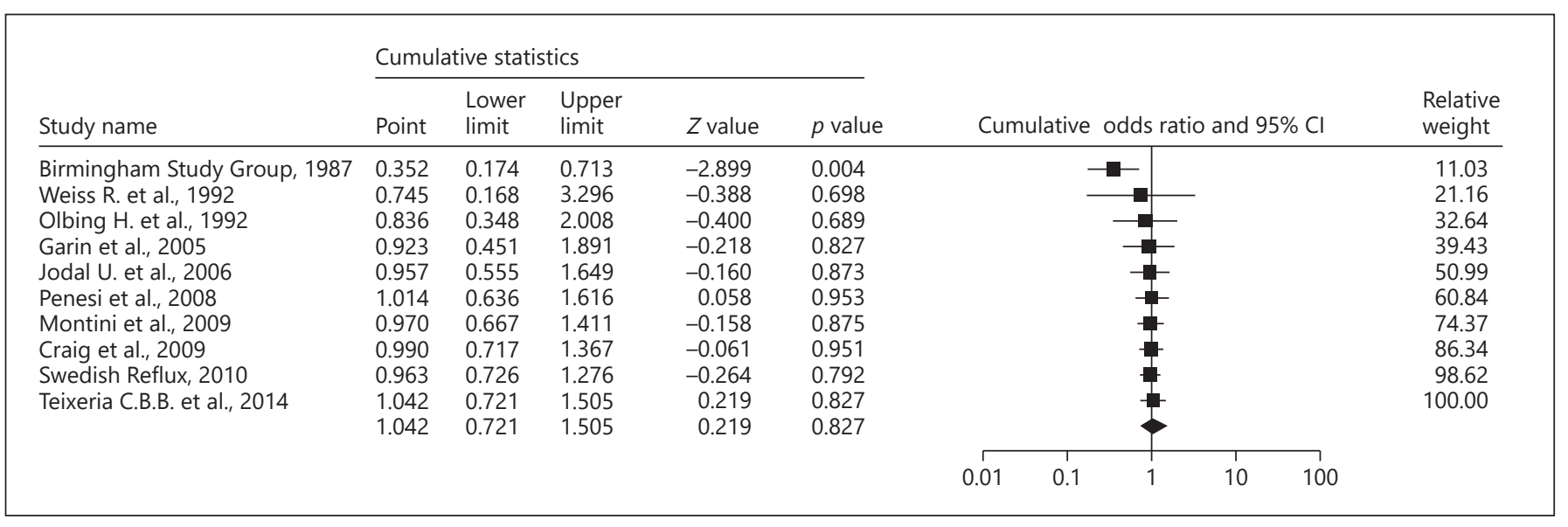

Fig. 5. Shows the forest plot for the renal scarring developed after treating VUR with surgery as compared to a conservative treatment approach. VUR, vesicoureteral reflux; OR, odds ratio.

Pyelonephritis after Endoscopic Surgery Compared to Antibiotics

The random-effects model was used to assess the 4 studies progressively for evaluating the odds of developing pyelonephritis in 2 groups, that is, surgery versus no surgery. The cumulative statistics showed a statistically significant $p<0.05$ under the random-effects model, with CI varying from 12.6 to $94.6 \%$. The point estimate (OR) was $<1(0.345)$, indicating that pyelonephritis is 0.345 less likely to occur after the surgical treatment of VUR compared to the conservative treatment approach (Fig. 6b).

\section{Discussion}

We looked at many articles, but only a couple of them were appropriate for further study, and none of them covered any of the scientifically relevant endpoints. Several trials focused on highly chosen patient samples, and the rest were historical, simply describing a collection of patients seen at the study institution. Treatment indications were not always given, and the patient population was rarely identified.

Just a few publications separately published statistics on the sexes, and the outcomes (kidney problems or VUR resolution) were frequently reported in terms of the number of kidneys, making it difficult to conclude what happened to the patients. In addition, any experiment was difficult to compare due to 4 different systems for scoring VUR. During follow-up, many did not monitor the resolution of VUR, and some did not rate the seriousness of the UTIs. We did not see any significant improvements in research architecture over time, unlike Jodal et al. [29], and the total importance of the publications was modest. Thus, the meta-analysis' limited strength is attributed in part to the fact that only 5 studies meet the criterion for 


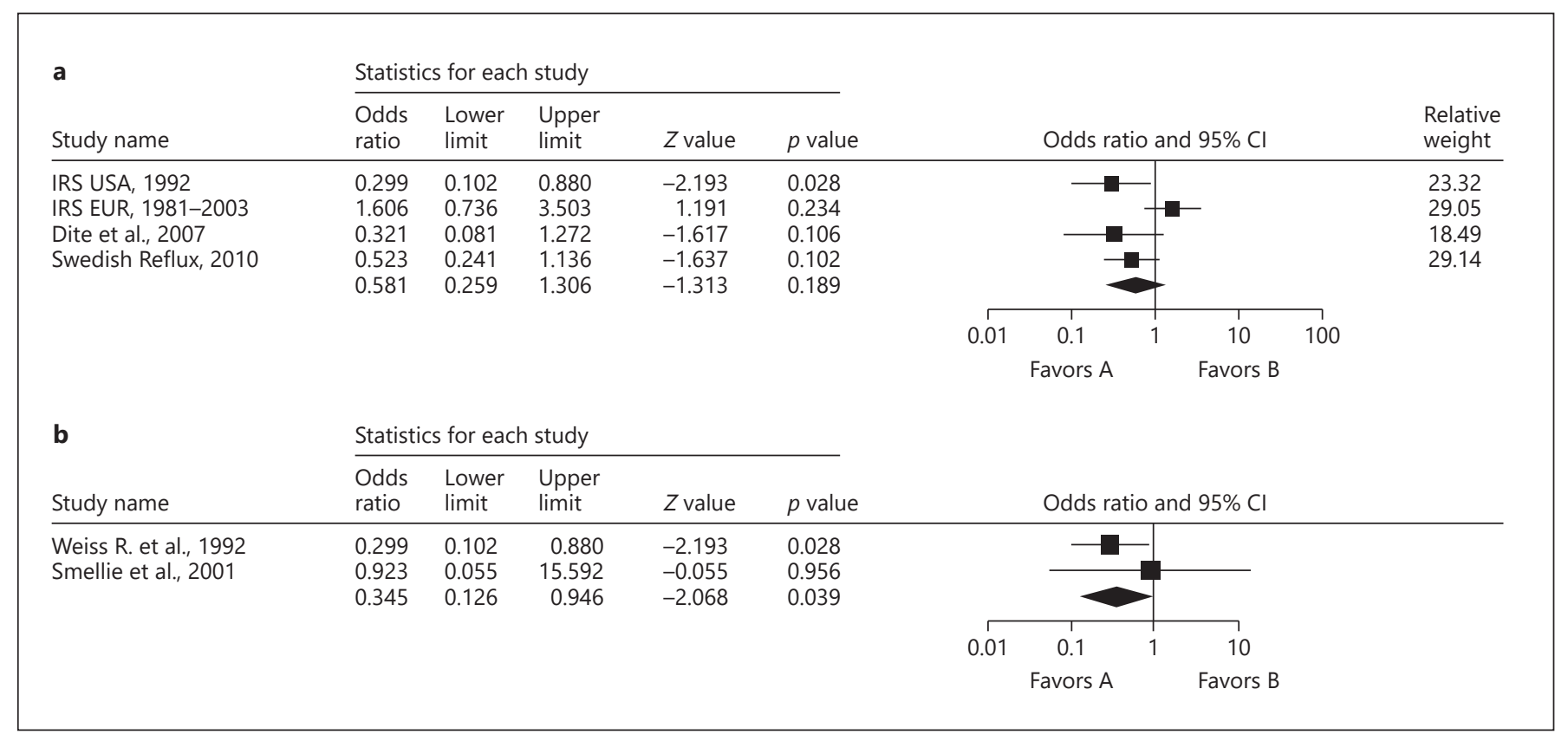

Fig. 6. a Shows the forest plot for the recurrent UTIs developed after treating VUR with surgery as compared to a conservative treatment approach. b Shows the forest plot for the pyelonephritis developed after treating VUR with surgery as compared to a conservative treatment approach. VUR, vesicoureteral reflux; OR, odds ratio; UTIs, urinary tract infections.

assessment, reflecting the unfortunate state of trials for the care of VUR in infants.

Antibiotic prophylaxis is most commonly used to treat recurrent UTIs among children with VUR based on the grading of VUR. However, the American Association of Pediatricians has issued guidelines to the community of pediatricians, suggesting that antibiotic prophylaxis may not be necessary and should be used judiciously to keep in mind the side effects of the same grading of VUR [3235]. Garin et al. [14], in their randomized controlled study among 236 patients, also found that urinary antibiotic prophylaxis does not affect the severity of UTIs in their patients with mild/moderate VUR. It was also seen by Montini et al. [5], wherein there is a greater risk of UTI in children of younger age after the antibiotic prophylaxis treatment for VUR. Brandström et al. [19], during the Swedish reflux trial in children, showed that $19 \%$ developed recurrent UTIs after antibiotic prophylaxis and $23 \%$ developed after surgical intervention in the treatment for VUR, which was 3 times less than in those children not receiving the prophylaxis. The RIVUR study involved 607 children (558 girls and 49 boys) with Grade I-IV VUR. Recurrent UTIs developed in 39 (13\%) out of 302 children who received prophylaxis, compared with 72 (24\%) out of 305 who received placebo (relative risk
0.55 , 95\% CI $0.38-0.78$ ) [20]. The present study also showed that the recurrent UTIs after antibiotic prophylaxis are lesser than that of the placebo group.

Continuous antibiotic prophylaxis is the most recommended treatment for correction of VUR. It consists of prescribing daily antibiotics at one-quarter to one-half the regular therapeutic dose. Trimethoprim-sulfamethoxazole, amoxicillin, and nitrofurantoin are the most commonly used CAP agents [36]. The present study shows that the chances of UTI after antibiotic treatment of VUR are less among pediatric patients than in a placebo group.

The surgical approach for treating VUR involves widening or changing the shape/angle of the ureter orifice for proper bladder filling via various techniques like open surgical ureteroneocystostomy, endoscopy, and laparoscopy. Hutch [37] first reported surgical correction in $77.7 \%$ of patients with VUR in 1952. Our meta-analysis shows that surgery successfully eliminates VUR, but there was no substantial difference between conservative and operative care in terms of recurrent UTIs, renal parenchymal defects, or renal scarring. Shannon and Feldman analyzed the findings of conservative and surgical care of VUR in a critical review of the literature. They concluded that, aside from eliminating reflux, surgery offered no 
other short-term benefits according to our study. Antibiotics are just a small part of the image [38].

Smellie et al. [6] do not support the hypothesis that surgical correction of VUR improves renal function in children with the bilateral disorder, which was confirmed by various studies on the same topic [9, 21-23]. Similarly, our meta-analysis indicates that a positive urine culture is more likely to develop in the surgery and antibiotics approach than antibiotics alone.

The presence of recurrent UTIs after the surgical correction of VUR was also reported by Dite et al. [31] and Brandström et al. [19], which indicated that patients with surgical intervention are 0.351 and 0.526 times more likely to develop recurrent UTIs than patients given conservative treatment. However, no statistical difference was noted in terms of recurrent UTIs to occur after surgical treatment or conservative approach for treating VUR as shown in this meta-analysis.

Weiss et al. [27] showed 0.742 times more likely to develop renal scarring and 0.299 times more likely to develop pyelonephritis after surgical treatment than conservative choice. Surgery does provide a significant advantage over pyelonephritis, according to all 4 RCTs included in our meta-analysis. As pyelonephritis is more likely to affect the kidneys than lower level UTIs, this may be taken to mean that surgery could not be favored for avoiding recurrences of UTI. However, even when using kidney injury as an endpoint, the supremacy of surgery was not shown.

Surgery for VUR is an extensive procedure that may cause problems $[12,14,29,39]$, prompting doctors to look for alternative treatments. Our meta-analysis found that conservative management (except for the elimination of VUR) is similarly efficient and that when children are followed for 10 years, $>70 \%$ of cases of dilating VUR can be resolved or increase dramatically with development $[6,7]$. Antimicrobials, especially nitrofurantoin, have been shown to cause gastrointestinal side effects in children aged $<2$ years, with up to one-third of these infants discontinuing prophylaxis [8].

Endoscopic therapy could be a safe option for these cases, although rates of VUR correction with endoscopic procedures are lower than those recorded with open reimplantations [40]. It has also been noted that parents prefer antimicrobial prophylaxis in the care of VUR over either endoscopic or open surgical treatment. Also, 5 years of antimicrobial prophylaxis over open surgery and 3 years of prophylaxis over endoscopic correction of VUR seem to be preferred by parents of children with VUR [41].

VUR and Adverse Effects
The meta-analysis has multiple limitations like the articles did not focus on secondary VUR. Included studies have not considered the side effects of treatment options for correction of VUR on its grade, and no limiting factor based on the year of publication has been considered. There is no difference reported in surgical than that in conservative treatment in terms of recurrent UTIs, renal scarring, and renal parenchymal defects.

\section{Conclusion}

Based on the findings of our meta-analysis, we believe that conservative care is appropriate for the vast majority of children with VUR and that such children should be tracked for several years before undergoing corrective surgery. In addition, surgical correction of VUR should be treated if there are repeated breakthrough UTIs or if prophylactic antimicrobials are not accepted.

\section{Statement of Ethics}

All procedures performed in studies involving human participants were in accordance with the ethical standards of the institutional and/or national research committee of University of Electronic Science and Technology of China, Children's Hospital of Shanxi, the Third Affiliated Hospital of Zhengzhou University, and Peking University First Hospital with the reference number PU \# RC/IRB/2020/1036 and with the 1964 Helsinki declaration and its later amendments or comparable ethical standards.

\section{Conflict of Interest Statement}

The authors have no conflicts of interest to declare.

\section{Funding Sources}

The authors did not receive any funding for this study.

\section{Author Contributions}

M.X. contributed to the concept and designed the study, X.X. analyzed data; Z.C. collected the data and helped in data analysis; H.X. contributed to drafting of the manuscript.

\section{Data Availability Statement}

The data used to support the findings of this study are available from the corresponding author upon request. 


\section{References}

1 Elder JS, Peters CA, Arant BS Jr, Ewalt DH, Hawtrey CE, Hurwitz RS, et al. Pediatric vesicoureteral reflux guidelines panel summary report on the management of primary vesicoureteral reflux in children. J Urol. 1997 May; 157(5):1846-51.

2 Hellström A, Hanson E, Hansson S, Hjälmås $\mathrm{K}$, Jodal U. Association between urinary symptoms at 7 years old and previous urinary tract infection. Arch Dis Child. 991;66:232-4.

3 Vitko D, Cho PS, Kostel SA, DiMartino SE, Cabour LD, Migliozzi MA, et al. Characterizing patients with recurrent urinary tract infections in vesicoureteral reflux: a pilot study of the urinary proteome. Mol Cell Proteomics. 2020;19:456-66.

4 Martinell J, Claesson I, Lidin-Janson G, Jodal U. Urinary infection, reflux and renal scarring in females continuously followed for 13-38 years. Pediatr Nephrol. 1995;9:131-6.

5 Montini G, Toffolo A, Zucchetta P, Dall'Amico R, Gobber D, Calderan A, et al. Antibiotic treatment for pyelonephritis in children: multicentre randomised controlled non-inferiority trial. BMJ. 2007;335(7616): 386.

6 Smellie JM, Prescod NP, Shaw PJ, Risdon RA, Bryant TN. Childhood reflux and urinary infection: a follow-up of 10-41 years in 226adults. Pediatric Nephrol. 1998 Nov;12: $727-36$.

7 Wennerstrom M, Hansson S, Jodal U, Sixt R, Stokland E. Renal function 16 to 26 years after the first urinary tract infection in childhood. Arch Pediatr Adolesc Med. 2000;154:339-45.

8 Wennerström M, Hansson S, Hedner T, Himmelmann A, Jodal U. Ambulatory blood pressure 16-26 years after the first urinary tract infection in childhood. JHypertens. $2000 \mathrm{Apr}$; 18:485-91.

9 Capozza N, Caione P. Dextranomer/hyaluronic acid copolymer implantation for vesico-ureteral reflux: a randomized comparison with antibiotic prophylaxis. J Pediatr. 2002 Feb;140(2):230-4.

10 Oswald J, Riccabona M, Lusuardi L, Bartsch G, Radmayr C. Prospective comparison and 1-year follow-up of a single endoscopic subureteral polydimethylsiloxane versus dextranomer/hyaluronic acid copolymer injection for treatment of vesicoureteral reflux in children. Urology. 2002;60:894-8.

11 Frankenschmidt A, Katzenwadel A, Zimmerhackl LB, Sommerkamp H. Endoscopic treatment of reflux by subureteric collagen injection: critical review of 5 years' experience. J Endourol. 1997;11:343-8.

12 Frey P, Gudinchet F, Jenny P. GAX 65: new injectable cross-linked collagen for the endoscopic treatment of vesicoureteral reflux: a double-blind study evaluating its efficiency in children. J Urol. 1997;158(3 Pt 2):1210-2.

13 Reddy PP, Evans MT, Hughes PA, Dangman B, Cooper J, Lepow ML, et al. Antimicrobial prophylaxis in children with vesico-ureteral reflux: a randomized prospective study of continuous therapy vs intermittent therapy vs surveillance. Pediatrics. 1997;100(3S):555-6.

14 Garin EH, Olavarria F, Garcia Nieto V, Valenciano B, Campos A, Young L. Clinical significance of primary vesicoureteral reflux and urinary antibiotic prophylaxis after acute pyelonephritis: a multicenter, randomized, controlled study. Pediatrics. 2006;117:626-32.

15 Pennesi M, Travan L, Peratoner L, Bordugo A, Cattaneo A, Ronfani L, et al. Is antibiotic prophylaxis in children with vesicoureteral reflux effective in preventing pyelonephritis and renal scars? A randomized, controlled trial. Pediatrics. 2008;121:e1489-94.

16 Montini G, Rigon L, Zucchetta P, Fregonese F, Toffolo A, Gobber D, et al. prophylaxis after first febrile urinary tract infection in children? A multicenter, randomized, controlled, noninferiority trial. Pediatrics. 2008;122:1064-71.

17 Roussey-Kesler G, Gadjos V, Idres N, Horen B, Ichay L, Leclair MD, et al. Antibiotic prophylaxis for the prevention of recurrent urinary tract infection in children with low grade vesicoureteral reflux: results from a prospective randomized study. J Urol. 2008;179:674-9.

18 Craig JC, Simpson JM, Williams GJ, Lowe A, Reynolds GJ, McTaggart SJ, et al. Antibiotic prophylaxis and recurrent urinary tract infection in children. N Engl J Med. 2009;361:1748-59.

19 Brandström P, Esbjörner E, Herthelius M, Swerkersson S, Jodal U, Hansson S. The Swedish reflux trial in children: III. Urinary tract infection pattern. J Urol. 2010 Jul;184:286-91.

20 Hoberman A, Chesney RW; RIVUR Trial Investigators. Antimicrobial prophylaxis for children with vesicoureteral reflux. N Engl J Med. 2014 Sep;371:1072-3.

21 Holland NH, Kazee M, Duff D, McRoberts JW. Antimicrobial prophylaxis in children with urinary tract infection and vesicoureteral reflux. Rev Infect Dis. 1982;4:467-74.

22 BIRSG. Prospective trial of operative versus nonoperative treatment of severe vesicoureteric reflux in children: five years' observation. Birmingham Reflux Study Group. Br Med J Clin Res. 1987;295(6592):237-41.

23 Morris MC, Rothwell DL, Paykel AD. A prospective study of vesico-ureteric reflux and renal function in children. 2nd CJ Hodson Symposium on Reflux Nephropathy; 1991.

24 Duckett JW, Walker RD, Weiss R. Surgical results: International Reflux Study in childrenUnited States branch. J Urol. 1992;148(5 Pt 2): 1674-5.

25 Smellie JM, Barratt TM, Chantler C, Gordon I, Prescod NP, Ransley PG, et al. Medical versus surgical treatment in children with severe bilateral vesicoureteric reflux and bilateral nephropathy: a randomised trial. Lancet. 2001; 357(9265):1329-33.

26 Ausgabe A. Comparison of conservative and surgical treatment of the vesico-uretero-renal reflux. International prospective reflux study in children. Urologe. 1981;20(2):107-15.
27 Weiss R, Duckett J, Spitzer A. Results of a randomized clinical trial of medical versus surgical management of infants and children with grades III and IV primary vesicoureteral reflux (United States). J Urol. 1992 Nov;148(5 Pt 2):1667-73.

28 Olbing H, Claësson I, Ebel KD, Seppänen U, Smellie JM, Tamminen-Möbius T, et al. Renal scars and parenchymal thinning in children with vesicoureteral reflux: a 5-year report of the International Reflux Study in children (European branch). J Urol. 1992;148(5 Pt 2):1653-6.

29 Jodal U, Smellie JM, Lax H, Hoyer PF. Tenyear results of randomized treatment of children with severe vesicoureteral reflux. Final report of the International Reflux Study in children. Pediatr Nephrol. 2006;21:785-92.

30 Teixeira CB, Cançado MA, Carvalhaes JT. Primary vesicoureteral reflux: conservative therapy or surgical intervention. J Bras $\mathrm{Ne}$ frol. 2014;36:10-7.

31 Dite Z, Kocvara R, Dvorácek J, Langer J, Sedlácek J. Endoscopic and conservative treatment of vesicoureteric reflux. Cas Lek Cesk. 2007;146(10):817-21.

32 Roberts KB; Subcommittee on Urinary Tract Infection; Steering Committee on Quality Improvement and Management. Urinary tract infection: clinical practice guideline for the diagnosis and management of the initial UTI in febrile infants and children 2 to 24 months. Pediatrics. 2011 Sep;128(3):595-610.

33 Finnell SM, Carroll AE, Downs SM; Subcommittee on Urinary Tract Infection. Technical report diagnosis and management of an initial UTI in febrile infants and young children. Pediatrics. 2011 Sep;128:e749-70.

34 Wan J, Skoog SJ, Hulbert WC; Executive Committee, Section on Urology, American Academy of Pediatrics. Section on urology response to new guidelines for the diagnosis and management of UTI. Pediatrics. 2012;129(4):e1051-3.

35 Mori R, Lakhanpaul M, Verrier-Jones K, et al. Diagnosis and management of urinary tract infection in children: summary of NICE guidance. BMJ. 2007 Aug 25;335(7616):395-7.

36 Johnston DL, Qureshi AH, Irvine RW, Giel DW, Hains DS. Contemporary management of vesicoureteral reflux. Curr Treat Options Pediatr. 2016;2:82-93.

37 Hutch JA. Vesico-ureteral reflux in the paraplegic: cause and correction. J Urol. 2002;167: 1410-4; discussion 1422.

38 Shannon A, Feldman W. Methodologic limitations in the literature on vesicouretral reflux: a critical review. J Pediatr. 1990;117:171-8.

39 Hodson EM, Wheeler DM, Vimalchandra D, Smith GH, Craig JC. Interventions for primary vesicoureteric reflux. Cochrane Database Syst Rev. 2007 Jul 18;(3):CD001532.

40 Läckgren G, Wåhlin N, Stenberg A. Endoscopic treatment of children with vesico-ureteric reflux. Acta Paediatr Suppl. 1999 Nov;88(431):62-71.

41 Ogan K, Pohl HG, Carlson D, Belman AB, Rushton HG. Parental preferences in the management of vesicoureteral reflux. J Urol. 2001;166:240-3. 